\title{
Intensive E-learning: a web-based educational tool for clinical practice guidelines and protocols in intensive care
}

Lone Krogh Kjaer, RN (intensive care), Clinical Supervisor, Intensive Care Unit, Aarhus University Hospital, Tage-Hansens Gade 2, 8000 Aarhus, Denmark.

Key words: Clinical practice guideline clinical protocol E -learning intensive care Internet training web-based

E-mail: lone.kjaer@oncable.dk

\section{SUMMARY}

-

Registered nurses newly employed in intensive care need supplementary guidance, especially during evening and night shifts.

- Intensive E-learning is an easy to use web-based clinical practice guideline together with a clinical protocol tool for assembling biomedical devices.

- Intensive E-learning is an application that is easy to expand, enabling clinical supervisors to develop it further, as required.

- $\quad$ The application is flexible and allows customisation and tailoring of guidelines to fit local clinical procedures and use of biomedical devices.

The author of this article is a clinical supervisor who developed the application in association with other nurse colleagues. and application of the Intensive E-learning tool.

\section{INTRODUCTION}

Intensive E-learning is a supplementary education tool that we have used in our intensive care unit (ICU) primarily for training and guidance of newly employed registered nurses. It is accessible 24 hours per day. It is an Internet-based clinical practice guideline and a clinical protocol tool that has been designed to assist nurses with the assembling of biomedical devices. The application is flexible and can be customised to conform to individual ICU guidelines and protocols and the wide variety of clinical procedures and biomedical devices that are used.

Intensive E-learning is a very simple application with 'flat' files linked in a traditional web page that was developed using the web-editor Microsoft ${ }^{\odot}$ Front Page ${ }^{\odot}$. The purpose of the application is to use a series of pictures with a few supplemental texts to develop a supportive and systematic approach to guide nurses through the assembling of biomedical devices or as a clinical practice guideline for complicated or infrequently used nursing procedures. It was important in the development of the application that it was easy and simple to work with, and that it could be further developed by the clinical supervisors in the department without the need for help from computer specialists. The application should not be confused with simulators or interactive multimedia

software applications with sound, image or video files or applications for authorisation or certification of nurses.

The topics for Intensive E-learning were chosen based on nurses' need for guidelines and instructions in clinical practice. In our ICU clinical practice guidelines are written for nursing procedures that are difficult to carry out or that are infrequently used and clinical protocols are written for biomedical devices that are difficult to assemble, new equipment that is to be introduced in the department and biomedical devices that are infrequently used. Many different procedures and protocols on assembling biomedical devices can be described and demonstrated using the Intensive E-learning tool.

\section{BACKGROUND}

The Aarhus Sygehus Tage-Hansens Gade ICU, in Aarhus, Denmark is a mixed clinical and surgical unit with six high level intensive care beds. The unit specialises in lower abdominal surgery, haematology and medicine. The nursing staff includes around thirty registered critical care nurses, ten registered nurses (RNs) and six social care and health care assistants.

In our ICU much effort is put into ensuring that new staff members are introduced to the various equipment and procedures in the unit. The introductory period of two months includes the opportunity for newly registered nurses to train in the use of most of the equipment, and to carry out some procedures; first in a laboratory setting and later in the clinical setting with supervision. Despite this, in the past, several newly employed RNs expressed their frustrations that they could not remember all of the necessary details when at a later time - perhaps during an evening or night shift - they had to use their knowledge independently. Unfortunately the limitations of our available resources do not allow a longer introductory period in our unit.

One of the problems was that some of the newly employed RNs found it difficult to ask a busy colleague for advice because they felt that they should already have mastered the biomedical devices and nursing procedures (since they had been trained in their use). Another problem was that some procedures are carried out very infrequently, for example, turning patients into the prone position, which is performed perhaps once a month. With 46 staff members it is difficult to keep everyone updated on procedures.

Our ICU of course has, as do many other ICUs, many well-writ- 


\section{* Intensive E-learning: a web-based educational tool for clinical practice guidelines and protocols in intensive care *}

ten clinical practice guidelines for all our procedures including complicated or infrequently used ones. However, they are not used very often because they are filed in large folders, it takes a long time to find them, and it can be difficult to locate specific details.

At a certain time in our unit, when there was a large turnover with a lot of new staff members and only a few clinical supervisors, it became clear that we had to take another approach to fulfilling the nursing staff's need for guidance and training, especially during evening and night shifts. So, we thought, why not exploit the fact that we have computers located in many places in our ICU? This was also relevant because we had observed that the younger nurses especially had a high computer literacy, and we felt that they would benefit from computer access for their learning needs.

In our ICU computers are easily accessible tools for the whole nursing team. They are placed close to each individual patient, and are central to the daily work routines. Using the computer as a guidance and instruction tool has the advantage that the user gets a very visual instruction compared to a written instruction. Pictures demonstrate an action better, and pictures can easily be accompanied with short written explanations. A combination of graphic and text-based material is very useful for demonstrating clinical practice guidelines as well as clinical protocols for assembling biomedical devices. In many respects the computer is a perfect tool; it is easy to use and can be accessed day and night, and allows the individual RN to handle different situations more independently.

The result of these considerations was that we decided to develop a set of visually based clinical practice guidelines together with clinical protocols for assembling biomedical devices. The application was named Intensive E-learning, since E-learning was, at that time, any form of computer-based instruction.

\section{DEVELOPING THE INTENSIVE E-LEARNING TOOL}

-

"How is this biomedical device assembled? I have been instructed once so it is kind of embarrassing asking my colleagues",

- $\quad$ "They are so busy and I don't want to interrupt and ask for help"

- $\quad$ "It has been quite a while since I carried out this procedure, I can hardly remember how to do it".

These are some of the statements that our clinical supervisors were confronted with when we began this project to develop an effective web-based Intensive E-learning tool that could be used in clinical practice.

Many ICUs spend a lot of time and many resources on teaching and guiding new staff, and RNs in ICU have to be capable of assembling many types of biomedical devices and handling a variety of clinical nursing procedures. Particularly for the new staff it can be very difficult to acquire the specific knowledge and working skills that are now provided by Intensive E-learning.

Intensive E-learning was developed using three commonly used software programs: Adobe Photoshop ${ }^{\odot}$; a picture editing program, Microsoft ${ }^{\odot}$ PowerPoint $^{\odot}$ and Microsoft ${ }^{\odot}$ Frontpage $^{\odot}$. The application is based on html/web programming and allows RNs with knowledge of the three software programs to develop an application for their specific needs in their own ICU. This means, that the application can be developed without the need for external computer specialists. This ensures that the rapid changes in tech- nology and the continuous need for updating nursing knowledge and competence can be met with an easy and fast update of the guidelines and the clinical protocols.

\section{USING THE TOOL TO DESCRIBE A PROCEDURE OR PROTOCOL}

A procedure/protocol is set up in the following way:

- $\quad$ Pictures are taken with a digital camera to show step-by-step how a biomedical device is assembled or how a nursing procedure is carried out.

- $\quad$ All steps are clearly shown and important, critical details that are known to cause problems are emphasised.

- $\quad$ Biomedical devices are photographed with a clear background in order to avoid distracting elements. Similarly, each step in a clinical practice guideline is photographed simply, avoiding distracting elements. The pictures are then edited using the photo editing software program. The important details are selected and the pictures are scaled to web-publishing format (reduced in size).

- $\quad$ A series of slides are developed using PowerPoint ${ }^{\circledR}$ (because it is easy to insert pictures and add accompanying explanations and comments). The text is educational and instructive and appears as a short but clarifying explanation with each picture (see Figure 1).

- $\quad$ The PowerPoint $@$ slide show is saved in a web publishing format, so that the procedure can be viewed in a web browser. To make each clinical protocol or clinical practice guideline easily accessible a web page was developed using Frontpage $^{\circledR}$, which has explanatory pictures and links to the procedures (see Figure 2).

- When a clinical practice guideline or a clinical protocol has been developed fully it requires approval by the Head of the ICU after which it is installed on all computers in the unit. (See Figure 3 for a complete example of a clinical procedure.)

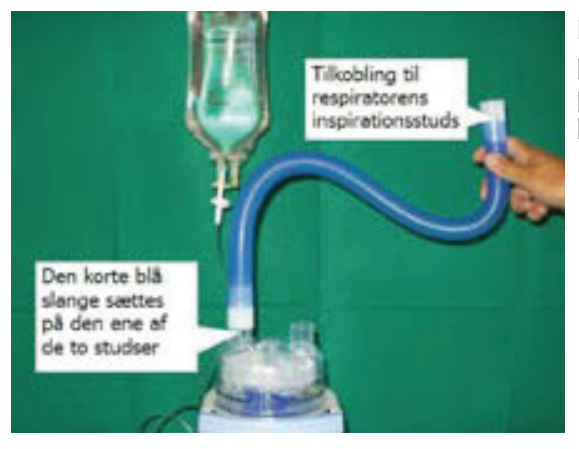

Figure 1. Example of picture from a clinical protocol for assembling biomedical devices

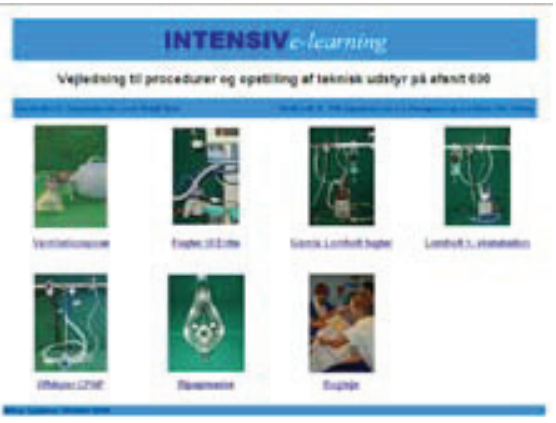

Figure 2. The front page, with different links 
* Intensive E-learning: a web-based educational tool for clinical practice guidelines and protocols in intensive care *

Slide 1

Slide 3

Slide 5

Slide 7

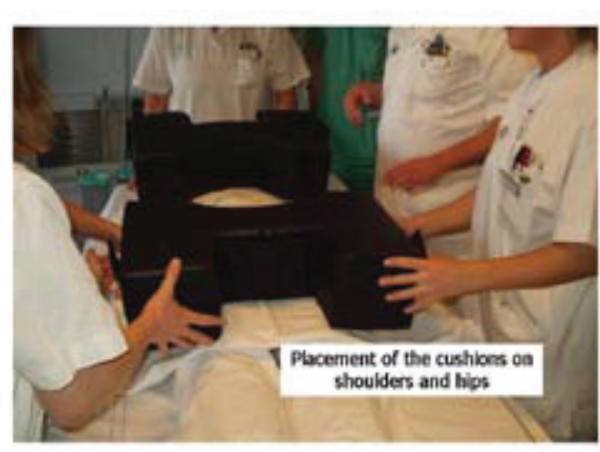

Slide 9

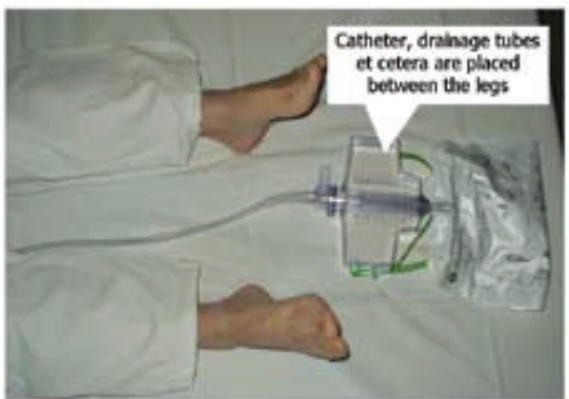

\section{Turning a patient into} the prone position

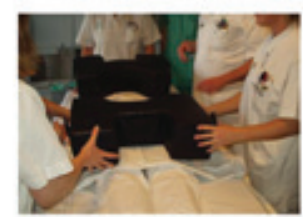

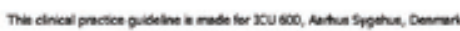

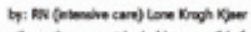

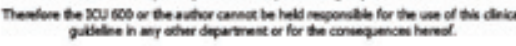
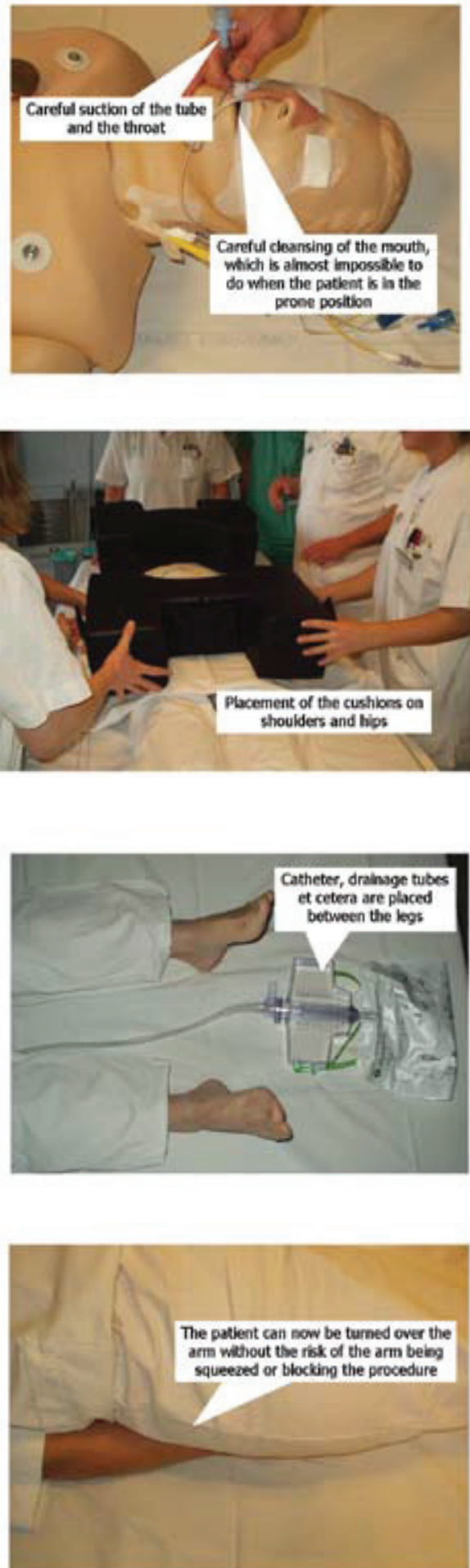

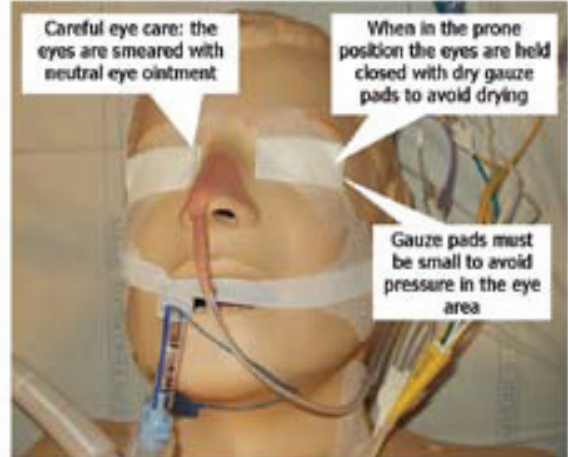

Slide 2

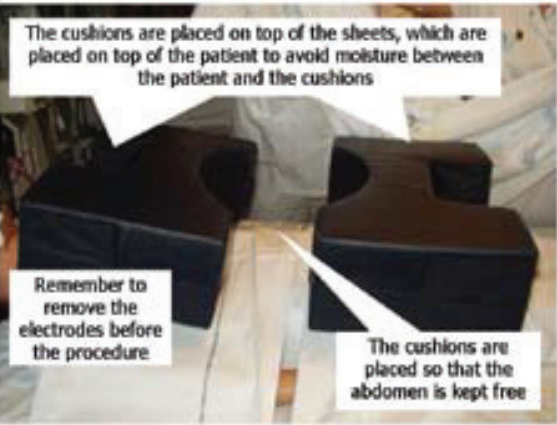

Slide 4

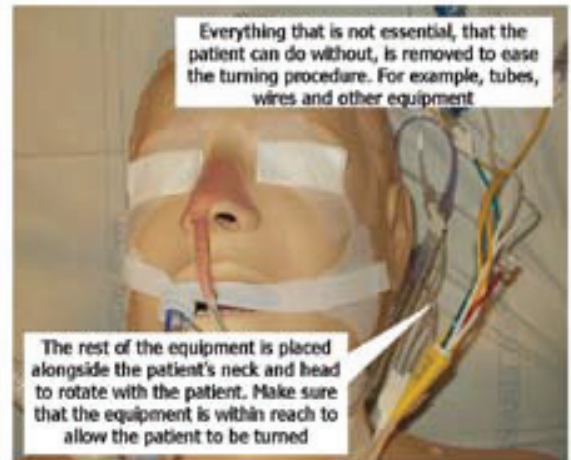

Slide 6

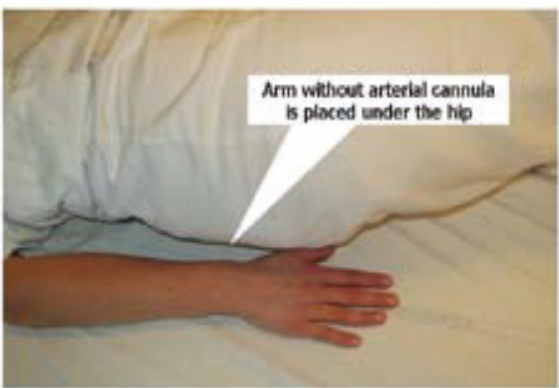

Slide 8

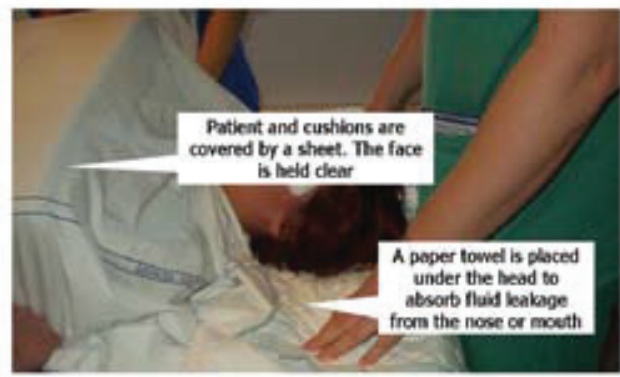

Slide 10 
Slide 11

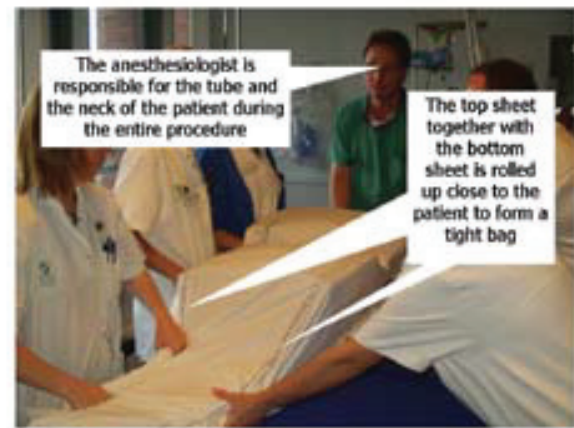

Slide 13

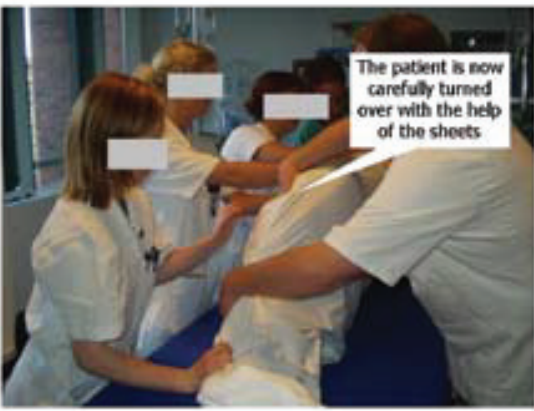

Slide 15

Slide 17
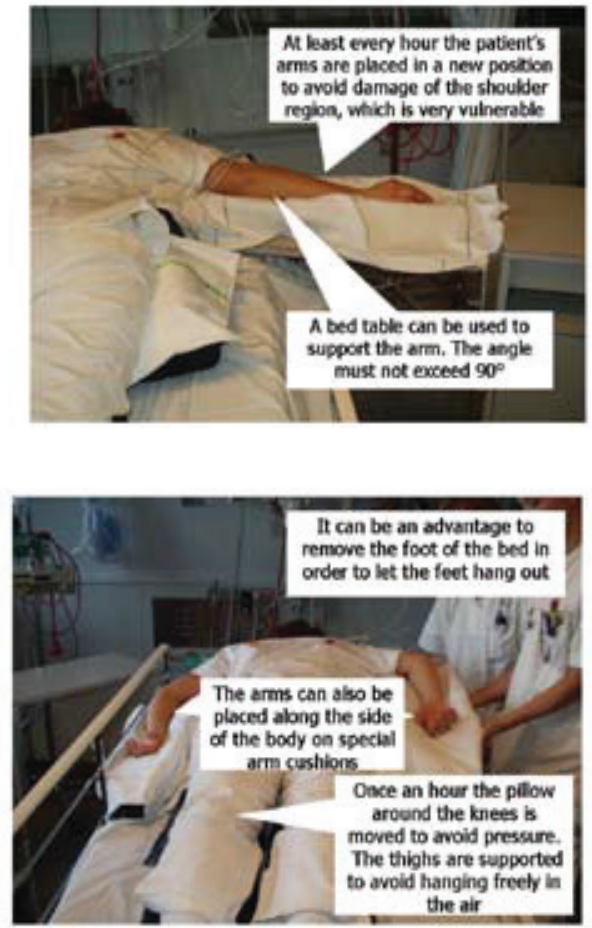

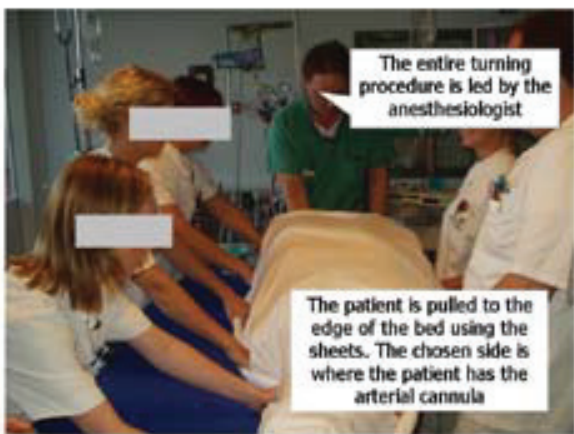

Slide 12

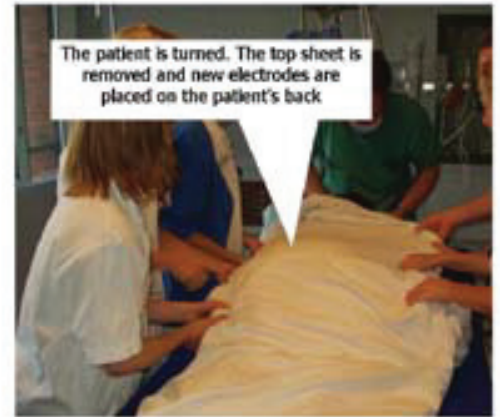

Slide 14

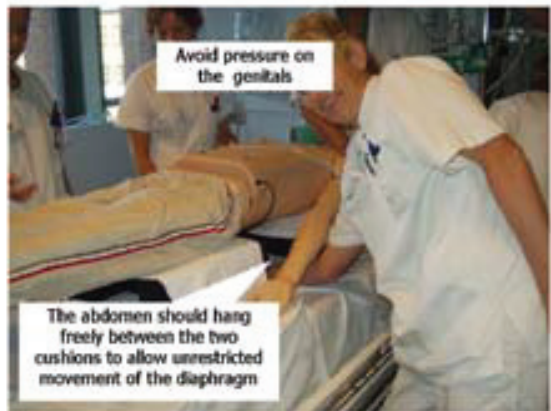

Slide 16

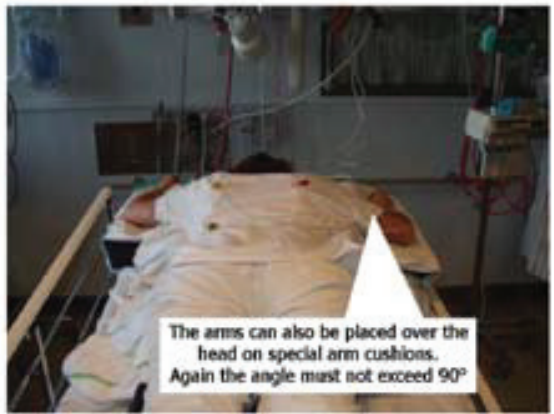

Slide 18

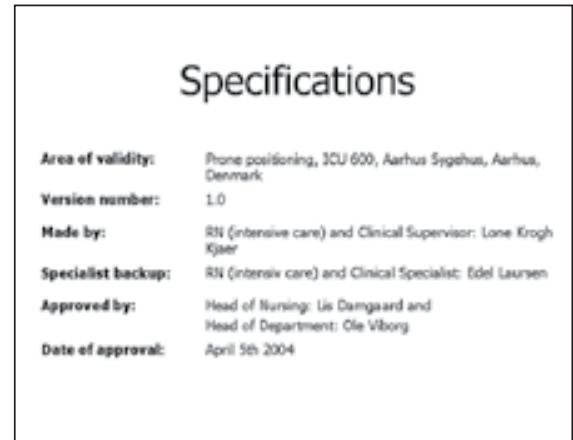

Slide 20 
USUNG THE INTENSIVE E-LEARNING TOOL IN CLINICAL

\section{PRACTICE}

The Intensive E-learning tool was presented to all the staff in the unit; however our experience was that specific training was not necessary because the system was so easy to use. The program is now used by all members of the nursing staff, whether they are about to carry out a procedure in which they are less experienced or if they just need to refresh their knowledge.

It has been a positive experience that the registered nurses have requested that more clinical practice guidelines and clinical protocols are added to the application. It has also been positive to see that the application has been used by our registered intensive care nurses to demonstrate and discuss different nursing procedures with newly employed staff. In the future we will need to extend the range of guidelines and protocols in the application, so that it becomes an even more relevant tool for nurses to use to maintain their knowledge and clinical competence.

It is too early to draw conclusions regarding the specific effects of using this e-learning application but it would be interesting to audit its use; who uses it, how many times is it used, and how effective it is with respect to ensuring the correct use of biomedical devices and implementation of nursing procedures. It is also important to ensure that all protocols and procedures are kept upto-date.

Finally, it is important to stress that Intensive E-learning cannot stand alone as an educational tool and it should be seen as supplementary to other more traditional approaches to clinical education. However it is unique in that it is a tool that can be accessed 24 hours per day, and its use of photographic illustrations makes it 'user-friendly' and easy to use for all members of staff. 